\title{
SENILE BLEPHAROSPASM
}

BY

\author{
K. E. Madan, M.D., D.O.M.S.
}

HONORARY OPHTHALMIC SURGEON, PARSEE GENERAL HOSPITAL, BOMBAY

Amongst the troublesome chronic diseases, like nystagmus and conical cornea, which are not generally satisfactorily benefited by treatment, are some cases of idiopathic persistent senile blepharospasm. This condition is quite different from the functional type, which can be easily diagnosed from the history, signs, and symptoms, and so can be treated; but the senile variety mostly shows itself as a clonic type of spasm causing frequent winking movements of the lids and inability to keep the eye fully open during the spasms, which may either affect the orbicularis palpebrarum only, or also the zygomatici, or may be a part of general facial spasm (tic convulsif). It is in these localized forms in which, if the underlying cause be made out as is possible in the symptomatic variety, the spasms would readily respond to treatment, but for senile blepharospasm no one cause is generally to be found.

Such cases are a source of difficulty to the ophthalmic surgeon and make him painfully conscious of his inability to cure the patient. However the following case in which some success was achieved and a cure almost occurred seems interesting and worthy of note :

D.B., a man, aged 56 years, had a cataract extraction done in his left eye five years previously. He came to me with the complaint that his vision after the operation was fairly good, but gradually dimmed and that near work could not be done, on account of which he seemed to be in great mental anxiety, and was suffering from clonic blepharospasm of this left eye, which he said came on some months after the extraction. Examination revealed that he had only monocular vision, as his right eye was blind, and that he wore $+13 \mathrm{D}$. sph. before his left eye, with which he saw $6 / 60$, and used to turn his head very slightly sideways to see clearly. Any other correction did not materially improve him for distant or for near sight.

Remnants of the capsular membrane were seen lying more in the central than in the peripheral part of the pupil. Another point that attracted attention was the condition of the skin of the lower lid and of the infra-orbital region, which was very lax and baggy. The patient stated that he had a feeling of heaviness in the lids of his left eye, and was conscious of the winking spasms whenever they occurred, as at that time he was unable to keep his eye even 
half open, and so he shunned society. I decided to improve his vision by a needling operation; I made a good central hole in the tough capsule, and the man was able to read J.1 with proper correction, and his distant vision also considerably improved, and became more distinct than before. His mental anxiety being quite relieved as now he was able to do his usual clerical work, the spasms were also fortunately found to be considerably reduced in their intensity and frequency, and according to the patient reduced by 50 per cent.; this is probably due to the fact that the opaque membranes of the capsule lying in the centre of the pupil obstructed the entrance of rays of light and refracted them irregularly, and thus may have reflexly caused the spasms. This is likely to be the case, for if only one of the two eyes is working, and if in this there is some sort of obstruction to clear vision, reflexes of different kinds may be set up by efforts of the patient to see clearly. He does not now turn his head sideways to see distant objects.

To endeavour to help the patient in getting rid of the spasms altogether electrical treatment was tried for some time, but without benefit. As there was much redundant loose skin in the infraorbital region I applied horizontally strips of adhesive plaster, with the idea of keeping the loose skin tightly stretched over the infra-orbital region; this seemed to be liked by the patient, as he said the twitching movements were less frequent and feebler than before. Every few days as the strips got dirty they were changed and so he kept on for some months during which he was also treated medicinally.

In the endeavour to attain a permanently satisfactory result I removed an oval piece of skin one inch broad and one and a half inches long on the outer side of the malar prominence and stitched up the edges; the skin then remained definitely stretched and the bagginess disappeared. The patient said he gradually felt better and almost cured of the troublesome spasms. Hence in this case it appears that the causes of blepharospasm were:

1. Extreme mental anxiety on account of not being able to do his clerical work, and the fear of losing his livelihood, on account of which he felt very nervous and passed sleepless nights.

2. Presence of dense capsular membrane in the central part of the pupil.

3. Loose baggy folds of skin of the lower eyelid. 